\title{
RAZONES A FAVOR DE UNA POSTMODERNIDAD ALTERNATIVA (RESPUESTA A JAVIER DE LUCAS)
}

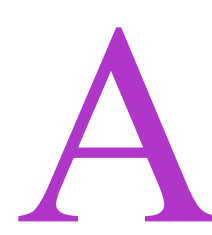

gradezco a Javier de Lucas su inteligente y amable comentario crítico a mi libro, Postmodernidad: decadencia o resistencia, que me ha ayudado a repensar a fondo sus tesis fundamentales y a revisar alguno de los puntos allí expuestos.

Javier de Lucas ha captado en mi opinión con toda claridad los dos rasgos esenciales del libro: de un lado, su inconformismo, motivado básicamente por la oposición a la persistencia en la sociedad actual de las dos notas dominantes de la modernidad: el individualismo y el economicismo, y de otro, su carácter esperanzado, fundada en la apertura a la transcendencia, desde la perspectiva del pensamiento cristiano. Javier de Lucas coincidiría en mi opinión con el análisis crítico y con la esperanza, pero consideraría que en el libro ha habido un exceso en la dosificación de ambas realidades. Al mismo tiempo discreparía en la conveniencia de la utilidad del rótulo «postmodernidad» para designar el nuevo paradigma que debería sustituir a la modernidad hegemónica, de carácter tecnocrático. Por ello me parece que el mejor modo de responder a su comentario crítico es hacer un balance de las razones del descontento respecto al presente y de la esperanza respecto al futuro, así como justificar el porqué de la conveniencia de seguir utilizando dicho rótulo de modo distinto al habitual que lo hace sinónimo de postestructuralismo.

1. Respecto al último tema, por el que empezaré, creo que son cada vez más numerosos los autores que utilizan el término postmodernidad, en un sentido semejante al utilizado en el libro que comentamos, esto es, en cuanto postmodernidad resistente, distinta de la decadencia y del irracionalismo de los posestructuralistas franceses. Así podría citarse, sin hacer referencia a los autores recogidos en el libro, a filósofos como Koslowski, Low, Groys, Muguerza o Sádaba, o a juristas, como Beck, Lombardi, B. Santos o R. Unger.

Lo adecuado del término, en mi opinión, radicaría en la exigencia de superar las dicotomías, heredadas del racionalismo 
cartesiano, y muy singularmente la disyuntiva entre libertad e igualdad, y en la necesidad de hacer hincapié en fraternidad-solidaridad, como integradora de ambas. En este sentido, los síntomas actuales de flexibilización de las fronteras creadas artificialmente en Yalta, y que habrían cristalizado hasta este momento la historia, pueden considerarse convergentes con las aspiraciones más profundas de la postmodernidad, en la medida en que impliquen una voluntad seria de acabar con los bloques, y no pura y simplemente de fortalecer uno de ellos. De ahí, a mi entender, lo totalmente incorrecto por el contrario del término «posthistoria», para calificar la situación actual. Lo que podemos estar asistiendo en el momento presente es a un desbloqueo de la historia, a una recuperación de la creatividad y movilidad sociales, al inicio, eso sí, de una nueva época histórica.

2. Por lo que se refiere al aspecto crítico, mi disconformidad radical con el capitalismo parte de la aceptación de la mayor eficiencia de dicho sistema para la producción de mercancías con el menor coste económico, o con el menor número de factores, y va dirigida precisamente contra la elevación de la eficiencia a criterio supremo de organización social, ya que ello comporta inevitablemente tres consecuencias:

a) La total amoralidad de las actividades humanas, en la medida en que el mérito del mercado parece consistir en transformar los vicios privados, como el egoísmo o la insolidaridad, en virtudes públicas, como el bienestar para todos.

b) El incremento de la dependencia respecto a la máquina, que crece paralelamente a la reducción de la dependencia de los individuos entre sí. (Sobre ello ha insistido con todo acierto Misham, en su clásico libro, sobre Los costes del crecimiento económico) (págs. 199 y sigs., y 215 y sigs.).

c) La sustitución de las necesidades por deseos, como móvil del comportamiento humano (Misham, pág. 38), ya que su lema es «lo suficiente no basta», y al mismo tiempo la negación de los derechos y obligaciones positivas. En ello consistiría para Hobsbahm (El mundo del trabajo, pág. 311), la innovación más espectacular, y a su juicio diabólica para la mayoría de las personas de la sociedad burguesa y su economía capitalista. Una versión reciente de este planteamiento capitalista la ofrece el libro de Peter Berger, que empeora sus planteamientos anteriores, al afirmar que el «derecho al desarrollo es una excrecencia de la mentalidad tercermundista» (La revolución capitalista, pág. 293 nota).

Por lo que respecta a las relaciones entre capitalismo y democracia habría que recordar que la única democracia apoyada 
desde el capitalismo ha sido la democracia política, esto es, aquella que hace referencia a la organización del Estado, y aún aquí se limita exclusivamente a la democracia representativa. Pero el capitalismo deja enteramente fuera del ámbito de la democracia tanto el campo de la economía interna a las fábricas, como el de las relaciones internacionales. (Sobre ello, se ha insistido en el pensamiento radical de Proudhon o Gurvitch a Santos.) Tal exclusión de la democracia y la participación en el ámbito de la economía conduce al incremento del paro estructural, que sólo con la reducción de la jornada laboral podría limitarse, y al endeudamiento y progresiva depauperación de los países vendedores de materias primas en favor de los vendedores de los productos manufacturados.

3. Por lo que se refiere al exceso de optimismo, creo que en el texto se habla tan sólo de tendencias más favorables a la resolución de los problemas que se están produciendo en los últimos tiempos, y que tendrían un carácter más positivo en lo político y en lo cultural que en lo económico (pág. 102 y sigs.).

Me parece además que los acontecimientos más recientes confirman esta esperanza en lugar de desmentirla. En el plano político, las razones para un mayor optimismo procederían del avance de la voluntad democratizadora, de lo que ha llamado A. Touraine «el agotamiento del modelo estatal voluntarista» (El País, 21-8-89). Este proceso sería especialmente visible en la Europa oriental, y resultaría especialmente estimulante en la medida en que no implicase una claudicación ante el modelo consumista de Occidente. Esta actitud en favor de nuevas perspectivas menos reduccionistas es la que parece implicar, por ejemplo, el discurso del primer ministro polaco, Tadeuzs Mazowiecki: «Tiene que surgir una nueva escala de valores y no sólo privilegiar la capacidad y el gozo del consumo: la preocupación de todos porque no se produzcan bolsas de pobreza, la promoción de una atmósfera favorable a los valores superiores a los puramente materiales» (Ya, 3-10-89).

El retorno de la democracia va unido claramente en este planteamiento, como en otros similares, como el Neues Forum de la RDA, a lo que C. S. Karol, ha llamado la «cultura de la resistencia», defensora de valores postmaterialistas, y por tanto podría entroncar con los movimientos alternativos, que también en Occidente defienden la primacía de la paz, y del respeto al medio ambiente. A su vez, esta irrupción de los valores postmaterialistas va íntimamente asociada a lo que para mí constituye otra de las razones de la esperanza, en este caso más directamente relacionadas con el terreno cultural: la recuperación del sentido 
de lo transcendente, que reaparece por doquier desde que Horkheimer glosó patéticamente la «nostalgia del Totalmente Otro».

El panorama europeo de los últimos tiempos parece caminar aunque sea muy lentamente hacia la construcción de la casa común, de los Urales al Atlántico, uno de los más bellos proyectos del fundador de la Perestroika, aunque no sea tan sólo un proyecto suyo, como se señala en el libro (pág. 123 y sig.). En cualquier caso, algo impensable hace bien poco, «la distensión Este-Oeste está haciendo que el gigantesco complejo militar-industrial comience a desmoronarse» (El País, 8-9-89).

4. Los acontecimientos en el Sur no parecen avalar el mismo optimismo, con el avance de los movimientos fundamentalistas, en los que la religión lejos de tener una orientación liberadora y humanista, presenta su rostro totalitario y fanático, así como con el incremento del hambre que explica la proliferación de las revueltas a menudo violentas. Habría por tanto razones que justificarían la afirmación de Javier de Lucas en el sentido de mi sobredosis de optimismo a la hora de juzgar la aportación de los países del Sur en la construcción de la nueva civilización. Creo, sin embargo, que se puede argumentar en favor de esta postura, destacando que los actuales planteamientos en favor de la reducción de tensiones entre los bloques y en favor del desarme podrían citar como precedentes importantes los textos de las conferencias internacionales de los países no alineados de Asia, África y Latino-América, que han tenido lugar desde la reunión de Bandung de 1955. ¿No fue precisamente este espíritu de distensión y pacifismo lo que llevó a utilizar a Sauvy por vez primera la expresión «Tercer Mundo», un año después de la Conferencia de Bandung, en sustitución de la expresión «naciones proletarias», empleada hasta entonces por Toynbee o P. Moussa?

De otro lado, mi simpatía por los pueblos del Tercer Mundo, y mi valoración en muchos aspectos positiva de su cultura, obedece, como ha visto con agudeza de Lucas, a mi identificación con el mundo de los humillados y ofendidos, que sería una de las razones de mi distanciamiento respecto a la Ilustración y a la filosofía habermasiana, ya que como se ha dicho recientemente, «en el seno de la cultura ilustrada es muy difícil experimentar la injusticia del sistema: la que explique nuestro bienestar desde nuestra relación con el Tercer Mundo... una solidaridad universal tiene que ser partisana» (R. Mate, Religión y socialismo. Más allá de la política). En esta actitud podrían converger pensadores tan dispares como Dostoievski, Bloch, S. Weil o Levinas.

5. Javier de Lucas ha visto que la justificación del capítulo 
dedicado al neofeminismo radica en constituir un síntoma más de la crisis del modelo de sociedad moderno-liberal, en que se basaba el primer feminismo. Pero no cree que este pretendido noefeminismo resulte compatible con otros temas señalados en el libro.

En mi opinión sí puede ser defendida esta convergencia, por cuanto si lo característico del primer feminismo consistiría en atribuir a la mujer la capacidad y derechos reservados hasta entonces a los varones, lo característico del neofeminismo consistiría en atribuir a los varones las obligaciones reservadas hasta ahora a las mujeres, como las relaciones con el cuidado por los más indigentes. De ahí la coincidencia de este neofeminismo con la preocupación pacifista por la no-violencia y con los movimientos ecologistas en la necesidad de reparto del tiempo de trabajo, también en el hogar, en definitiva la coincidencia en la experiencia de un «nuevo humanismo», que sustituya al moderno tecnocrático, agresivo, competitivo, en definitiva, viril.

Es cierto que en este feminismo postmoderno o radical, resulta más clara la insistencia en las obligaciones de no-discriminación y de no-violencia de los varones, y de ahí la exclusión total de la pornografía como violencia (sobre ello, Mac Kinnon, Feminism Unmodified), que la conciencia de las propias obligaciones de las mujeres en relación sobre todo con la maternidad. Es aquí donde surgen mayores divergencias, aunque parece irse abriendo paso la tesis que tiende a ver la legalización del aborto como machismo, es decir, como «extensión a las madres del más antiguo y brutal de los derechos patriarcales; el poder de disponer de la vida de los hijos sin restricciones» (J. Campos, El País, 10-10-89) (cfr. Virginia Held).

6. La inseparabilidad del derecho y el deber aparece por tanto como el rasgo más característico de la mentalidad postmoderna en lo que se refiere a la problemática de los derechos humanos, de ahí lo acertado de la afirmación de Javier de Lucas de la inexistencia de derechos de carácter absoluto, incluido el más fundamental de todos ellos, el derecho a la vida. Lo que define la inalienabilidad no es la total imposibilidad de renuncia del derecho por parte de su titular, cuanto la vinculación de tal posible renuncia al cumplimiento de un deber que va intrínsecamente incorporado al sentido de ese derecho, como es el caso de los supuestos certeramente señalados por De Lucas. Podría por tanto afirmarse que es precisamente por su carácter inalienable, por la conexión derecho-deber, por lo que los derechos no pueden considerarse absolutos es decir, ilimitados o carentes de vínculos. 
Me parece sin embargo que en mi libro debía haberse diferenciado mejor entre la simple inmoralidad de un comportamiento, como el del suicidio, en el que Kant advirtió la violación del imperativo categórico, y su antijuridicidad. Esta, en efecto, sólo aparece cuando se dan los daños directos contra terceros.

Por otro lado, quizás se debió establecer mejor la distinción entre el derecho subjetivo, como categoría técnico-jurídica, es decir, como pretensión capaz de ser ejercida ante los tribunales, cuyo traslado al ámbito internacional y no sólo interregional sería clave de bóveda del progreso jurídico, en materia de derechos humanos, y la proyección ideológica de dicho concepto, como absolutización de la pretensión, sin atención al correspondiente deber, que es lo que verdaderamente habría que superar en el tránsito de la sociedad moderna individualista a la postmoderna solidaria.

En el comentario crítico de Javier de Lucas hay muchas más sugerencias para continuar el debate, pero las tratadas por mí me han parecido las fundamentales. En todo caso, quedan ahí como incentivo para continuar dialogando en otra ocasión. 